\title{
Prediction of rehabilitation needs after treatment of cervical cancer: a methodological mistake
}

\author{
Siamak Sabour $^{1,2}$ (D) \\ Received: 11 February 2017 / Accepted: 10 April 2017 / Published online: 26 April 2017 \\ (C) Springer-Verlag Berlin Heidelberg 2017
}

I was interested to read the paper by Mikkelsen TB and colleagues published in Support Care Cancer March 2017. Women treated for cervical cancer with radiotherapy and chemotherapy have reported serious bowel, vaginal, and sexual late effects. Women, mean age 55 years, treated for cervical cancer from January 2010 to July 2013, who were alive and without known relapse/metastases were included in a cross-sectional study. The aim of the authors was to describe the late adverse effects, health-related quality of life, and selfefficacy in a representative Danish cervical cancer population in order to predict rehabilitation needs. Additionally, the authors reported that symptom experience was significantly higher in participants with locally advanced disease than in those with local disease. Self-efficacy was significantly lower in participants with locally advanced disease. The incidence of lymphedema was significantly higher among participants who were obese. The study suggested that young, obese survivors with locally advanced cervical cancer and survivors who received chemotherapy may have a serious risk of developing late adverse effects; thus, rehabilitation should target these needs [1].

However, this result has nothing to do with prediction. First, for prediction studies, we need data from two different cohorts or at least from one cohort divided into two to first to develop a prediction model and subsequently validate it.

Siamak Sabour

s.sabour@sbmu.ac.ir

1 Safety Promotion and Injury Prevention Research Center, Shahid Beheshti University of Medical Sciences, Tehran, Iran

2 Department of Clinical Epidemiology, School of Health, Shahid Beheshti University of Medical Sciences, Tehran, Iran
Misleading results are generally the main outcome of research that fails to validate its prediction models. Moreover, significantly higher or lower frequency of a symptom in a specified study population does not necessarily mean prediction value of the mentioned symptoms [2-6].

Finally, in prediction studies, we must assess the interactions between important variables. Final results can be impacted dramatically when qualitative interactions are present [2-6]. This means that most of the time, without assessing the interaction terms, prediction studies will mainly produce misleading messages.

\section{Compliance with ethical standards}

Conflict of interest The author declares that he has no conflict of interest.

\section{References}

1. Mikkelsen TB, Sørensen B, Dieperink KB (2017) Prediction of rehabilitation needs after treatment of cervical cancer: what do late adverse effects tell us? Support Care Cancer 25(3):823-831. doi: 10.1007/s00520-016-3466-x Epub 2016 Nov 10

2. Rothman KJ, Greenland S, Lash TL (2008) Cohort studies. In: Rothman KJ (ed) Modern Epidemiology, 3rd edn. Lippincott Williams \& Wilkins, Baltimore, pp 79-85

3. Sabour S (2015) Prediction of preterm delivery using levels of VEGF and leptin in amniotic fluid from the second trimester: prediction rules. Arch Gynecol Obstet 291(4):719. doi:10.1007/s00404-0143568-y Epub 2014 Dec 10

4. Sabour S (2015) Prediction of 3-dimensional pharyngeal airway changes after orthognathic surgery: a methodological issue. Am J Orthod Dentofac Orthop 147(1):8. doi:10.1016/j.ajodo.2014.10.016

5. Sabour S (2014) Obesity predictors in people with chronic spinal cord injury: a common mistake. J Res Med Sci 19(1):80

6. Sabour S, Ghassemi F (2013) Predictive value of confocal scanning laser for the onset of visual field loss. Ophthalmology 120(6):e31e32. doi:10.1016/j.ophtha.2013.01.055 